\title{
The Spread of Obesity in a Large Social Network Over 32 Years.
}

\section{Citation}

Christakis, Nicholas A. and James H. Fowler. 2007. The spread of obesity in a large social network over 32 years. The New England Journal of Medicine 357, no. 4: 370-379.

\section{Published Version}

http://dx.doi.org/10.1056/NEJMsa066082

\section{Permanent link}

http://nrs.harvard.edu/urn-3:HUL.InstRepos:3710802

\section{Terms of Use}

This article was downloaded from Harvard University's DASH repository, and is made available under the terms and conditions applicable to Other Posted Material, as set forth at http:// nrs.harvard.edu/urn-3:HUL.InstRepos:dash.current.terms-of-use\#LAA

\section{Share Your Story}

The Harvard community has made this article openly available.

Please share how this access benefits you. Submit a story.

\section{Accessibility}




\title{
The Spread of Obesity in a Large Social Network Over 32 Years
}

Nicholas A. Christakis, M.D., Ph.D., M.P.H., and James H. Fowler, Ph.D.

\author{
ABSTRACT
}

From the Department of Health Care Policy, Harvard Medical School, Boston (N.A.C.); the Department of Medicine, Mt. Auburn Hospital, Cambridge, MA (N.A.C.); the Department of Sociology, Harvard University, Boston (N.A.C.); and the Department of Political Science, University of California, San Diego, San Diego (J.H.F.). Address reprint requests to Dr. Christakis at the Department of Health Care Policy, Harvard Medical School, 180 Longwood Ave., Boston, MA 02115, or at christakis@hcp.med.harvard.edu.

N Engl J Med 2007;357:370-9.

Copyright $(2007$ Massachusetts Medical Society.

\section{BACKGROUND}

The prevalence of obesity has increased substantially over the past 30 years. We performed a quantitative analysis of the nature and extent of the person-to-person spread of obesity as a possible factor contributing to the obesity epidemic.

\section{METHODS}

We evaluated a densely interconnected social network of 12,067 people assessed repeatedly from 1971 to 2003 as part of the Framingham Heart Study. The bodymass index was available for all subjects. We used longitudinal statistical models to examine whether weight gain in one person was associated with weight gain in his or her friends, siblings, spouse, and neighbors.

\section{RESULTS}

Discernible clusters of obese persons were present in the network at all time points, and the clusters extended to three degrees of separation. These clusters did not appear to be solely attributable to the selective formation of social ties among obese persons. A person's chances of becoming obese increased by $57 \%$ ( $95 \%$ confidence interval [CI], 6 to 123) if he or she had a friend who became obese in a given interval. Among pairs of adult siblings, if one sibling became obese, the chance that the other would become obese increased by $40 \%$ ( $95 \%$ CI, 21 to 60). If one spouse became obese, the likelihood that the other spouse would become obese increased by $37 \%$ (95\% CI, 7 to 73). These effects were not seen among neighbors in the immediate geographic location. Persons of the same sex had relatively greater influence on each other as compared with those of the opposite sex. The spread of smoking cessation did not account for the spread of obesity in the network.

\section{CONCLUSIONS}

Network phenomena appear to be relevant to the biologic and behavioral trait of obesity, and obesity appears to spread through social ties. These findings have implications for clinical and public health interventions. 
T HE PREVALENCE OF OBESITY HAS INcreased from $23 \%$ to $31 \%$ over the recent past in the United States, and $66 \%$ of adults are now overweight. ${ }^{1,2}$ Proposed explanations for the obesity epidemic include societal changes that promote both inactivity and food consumption. ${ }^{3}$ The fact that the increase in obesity during this period cannot be explained by genetics ${ }^{4,5}$ and has occurred among all socioeconomic groups ${ }^{1}$ provides support for a broad set of social and environmental explanations. Since diverse phenomena can spread within social networks, ${ }^{6-10}$ we conducted a study to determine whether obesity might also spread from person to person, possibly contributing to the epidemic, and if so, how the spread might occur.

Whereas obesity has been stigmatized in the past, attitudes may be changing. ${ }^{11,12}$ To the extent that obesity is a product of voluntary choices or behaviors, the fact that people are embedded in social networks and are influenced by the evident appearance and behaviors of those around them suggests that weight gain in one person might influence weight gain in others. Having obese social contacts might change a person's tolerance for being obese or might influence his or her adoption of specific behaviors (e.g., smoking, eating, and exercising). In addition to such strictly social mechanisms, it is plausible that physiological imitation might occur; areas of the brain that correspond to actions such as eating food may be stimulated if these actions are observed in others. ${ }^{13}$ Even infectious causes of obesity are conceivable. ${ }^{14,15}$

We evaluated a network of 12,067 people who underwent repeated measurements over a period of 32 years. We examined several aspects of the spread of obesity, including the existence of clusters of obese persons within the network, the association between one person's weight gain and weight gain among his or her social contacts, the dependence of this association on the nature of the social ties (e.g., ties between friends of different kinds, siblings, spouses, and neighbors) and their direction, and the influence of sex, smoking behavior, and geographic distance between the domiciles of persons in the social network.

\section{METHODS}

\section{SOURCE DATA}

The Framingham Heart Study was initiated in 1948, when 5209 people were enrolled in the orig- inal cohort. ${ }^{16}$ The Framingham Offspring Study began in 1971, when most of the children of members of the original cohort and their spouses were enrolled in the offspring cohort. ${ }^{17}$ There has been almost no loss to follow-up other than death in this cohort of 5124 people; only 10 people left the study. In 2002, the third-generation cohort, consisting of 4095 children of the offspring cohort, was initiated. All participants undergo physical examinations (including measurements of height and weight), and they complete written questionnaires at regular intervals.

\section{NETWORK ASCERTAINMENT}

For our study, we used the offspring cohort as the source of 5124 key subjects, or "egos," as they are called in social-network analysis. Any persons to whom the egos are linked - in any of the Framingham Heart Study cohorts - can, however, serve as "alters." Overall, 12,067 living egos and alters were connected at some point during the study period (1971 to 2003).

To create the network data set, we entered information about the offspring cohort into a computer. This information was derived from archived, handwritten administrative tracking sheets that had been used since 1971 to identify people close

\section{Glossary.}

Ego: The person whose behavior is being analyzed.

Alter: A person connected to the ego who may influence the behavior of the ego.

Node: An object that may or may not be connected to other objects in a network. In this study, nodes represent people in the Framingham Heart Study cohorts.

Tie: A connection between two nodes that can be either one way (directed) or two ways (bilateral). In this study, all family ties (e.g., between siblings, parents, and spouses) are bilateral, but friendship ties are directional since a subject may identify someone as a friend who does not necessarily identify that person as a friend in return.

Degree of separation: The social distance between two people as measured by the smallest number of intermediaries between an ego and other members of the network. For a given ego, alters are degree 1 , since they are directly connected to the ego. Nodes that are connected to the alters but not to the ego are degree 2 (alters' alters). Nodes that are connected to the alters' alters but not to the ego are degree 3 , and so on.

Homophily: The tendency for people to choose relationships with people who have similar attributes.

Induction: The spread of a behavior or trait from one person to another.

Cluster: A group of nodes, each of which is connected to at least one other node in the group.

Connected component: Part of a social network in which all persons have a social tie to at least one other person and no person is connected to a member of any other commponent. 
to the study participants in order to facilitate follow-up. These sheets contain valuable, previously unused social-network information because they systematically and comprehensively identify relatives and friends named by the ego. The tracking sheets provide complete information about all first-order relatives (parents, spouses, siblings, and children), whether they are alive or dead, and at least one "close friend" at each of seven examinations between 1971 and 2003. The examinations took place during 3-year intervals centered in 1973, 1981, 1985, 1989, 1992, 1997, and 1999.

Many of the named alters on these sheets also were members of Framingham Heart Study cohorts. Detailed home addresses were also recorded at each time point; we used this information to calculate the geographic distance between people. This newly computerized database thus identifies the network links among participants at each examination and longitudinally from one examination to the next. As a person's family changed because of birth, death, marriage, or divorce, and as contacts changed because of residential moves or new friendships, this information was recorded. Furthermore, dates of birth and death were available from separate Framingham Heart Study files.

Overall, there were 38,611 observed social and family ties to the 5124 egos, yielding an average of 7.5 ties per ego (not including neighbors). For example, $83 \%$ of the spouses of egos were directly and repeatedly observed at the time of examination, and $87 \%$ percent of egos with siblings had at least one sibling in the network. For 10\% of the egos, an immediate neighbor also participated in the study; more expansive definitions of neighbors yielded similar results.

A total of $45 \%$ of the 5124 egos were connected through friendship to another person in the network. There were 3604 unique, observed friendships, for an average of 0.7 friendship tie per ego. Because friendship identifications are directional, we studied three different kinds of friendships: an "ego-perceived friendship," in which an ego identifies an alter as a friend; an "alter-perceived friendship," in which an alter identifies an ego as a friend; and a "mutual friendship," in which the identification is reciprocal. We hypothesized that a friend's social influence on an ego would be affected by the type of friendship, with the strongest effects occurring in mutual friendships, followed by ego-perceived friendships, fol- lowed by alter-perceived friendships. Our reasoning was that the person making the identification esteems the other person and may wish to emulate him or her.

We included only persons older than 21 years of age at any observation point and subsequently. At the inception of the study, $53 \%$ of the egos were women, the mean age of the egos was 38 years (range, 21 to 70), and their mean educational level was 13.6 years (range, no education to $\geq 17$ years of education).

The study data are available from the Framingham Heart Study. The study was approved by the institutional review board at Harvard Medical School; all subjects provided written informed consent.

\section{STATISTICAL ANALYSIS}

We graphed the network with the use of the Kamada-Kawai ${ }^{18}$ algorithm in Pajek software. ${ }^{19}$ We generated videos of the network by means of the Social Network Image Animator. ${ }^{20}$ We examined whether our data conformed to theoretical network models such as the small-world, ${ }^{10}$ scalefree, ${ }^{21}$ and hierarchical types ${ }^{22}$ (see the Supplementary Appendix, available with the full text of this article at www.nejm.org).

We defined obesity as a body-mass index (the weight in kilograms divided by the square of the height in meters) of 30 or more. Analyses in which the body-mass index was a continuous variable did not yield different results.

We considered three explanations for the clustering of obese people. First, egos might choose to associate with like alters ("homophily"). ${ }^{21,23,24}$ Second, egos and alters might share attributes or jointly experience unobserved contemporaneous events that cause their weight to vary at the same time (confounding). Third, alters might exert social influence or peer effects on egos ("induction"). Distinguishing the interpersonal induction of obesity from homophily requires dynamic, longitudinal network information about the emergence of ties between people ("nodes") in a network and also about the attributes of nodes (i.e., repeated measures of the body-mass index). ${ }^{25}$

The basic statistical analysis involved the specification of longitudinal logistic-regression models in which the ego's obesity status at any given examination or time point $(t+1)$ was a function of various attributes, such as the ego's age, sex, and 
educational level; the ego's obesity status at the previous time (t); and most pertinent, the alter's obesity status at times $t$ and $t+1 .{ }^{25}$ We used generalized estimating equations to account for multiple observations of the same ego across examinations and across ego-alter pairs. ${ }^{26}$ We assumed an independent working correlation structure for the clusters. ${ }^{26,27}$

The use of a time-lagged dependent variable (lagged to the previous examination) eliminated serial correlation in the errors (evaluated with a Lagrange multiplier test ${ }^{28}$ ) and also substantially controlled for the ego's genetic endowment and any intrinsic, stable predisposition to obesity. The use of a lagged independent variable for an alter's weight status controlled for homophily. ${ }^{25}$ The key variable of interest was an alter's obesity at time $t+1$. A significant coefficient for this variable would suggest either that an alter's weight affected an ego's weight or that an ego and an alter experienced contemporaneous events affect- ing both their weights. We estimated these models in varied ego-alter pair types.

To evaluate the possibility that omitted variables or unobserved events might explain the associations, we examined how the type or direction of the social relationship between the ego and the alter affected the association between the ego's obesity and the alter's obesity. For example, if unobserved factors drove the association between the ego's obesity and the alter's obesity, then the directionality of friendship should not have been relevant.

We evaluated the role of a possible spread in smoking-cessation behavior as a contributor to the spread of obesity by adding variables for the smoking status of egos and alters at times $t$ and $\mathrm{t}+1$ to the foregoing models. We also analyzed the role of geographic distance between egos and alters by adding such a variable.

We calculated $95 \%$ confidence intervals by simulating the first difference in the alter's contem-

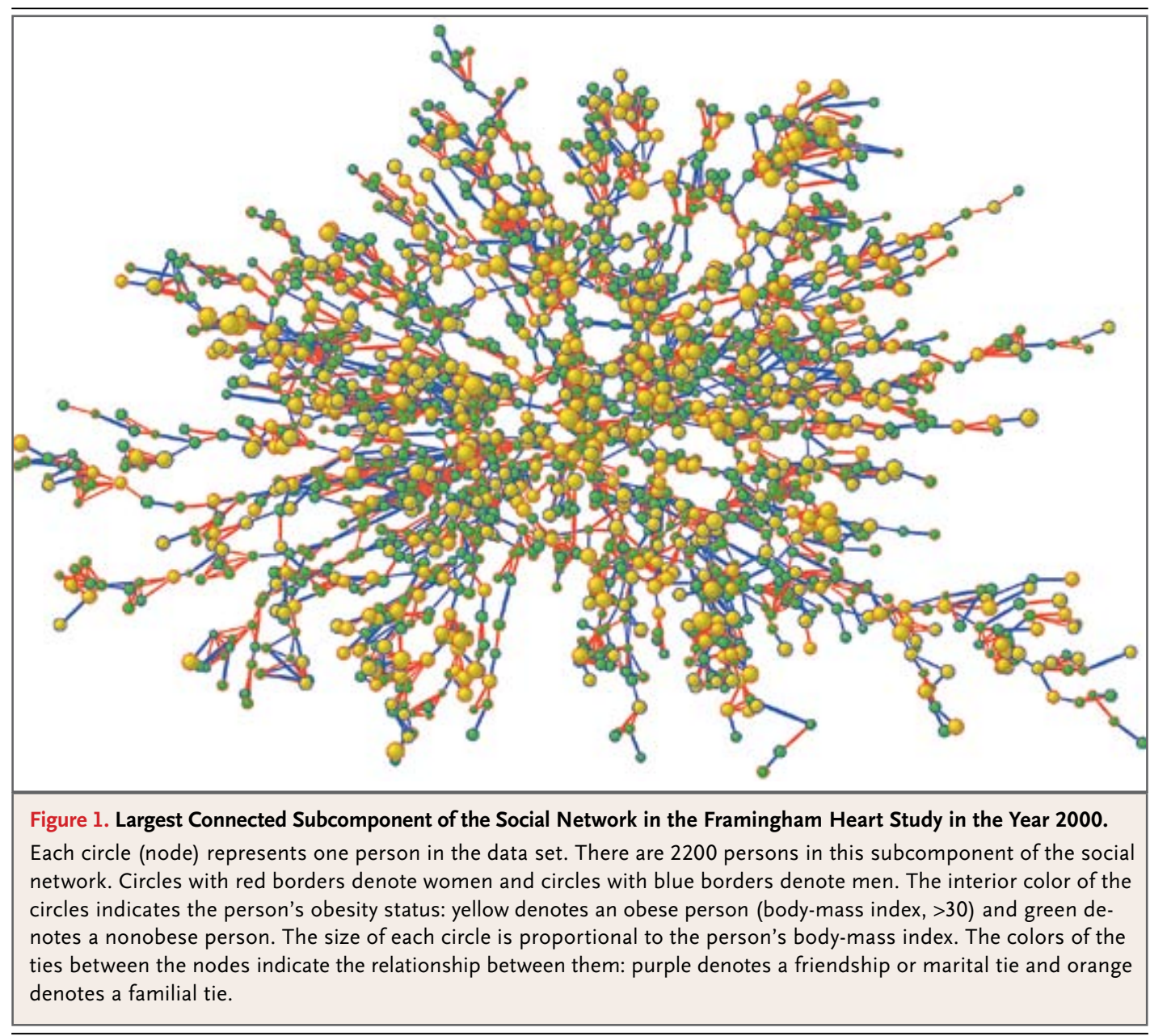




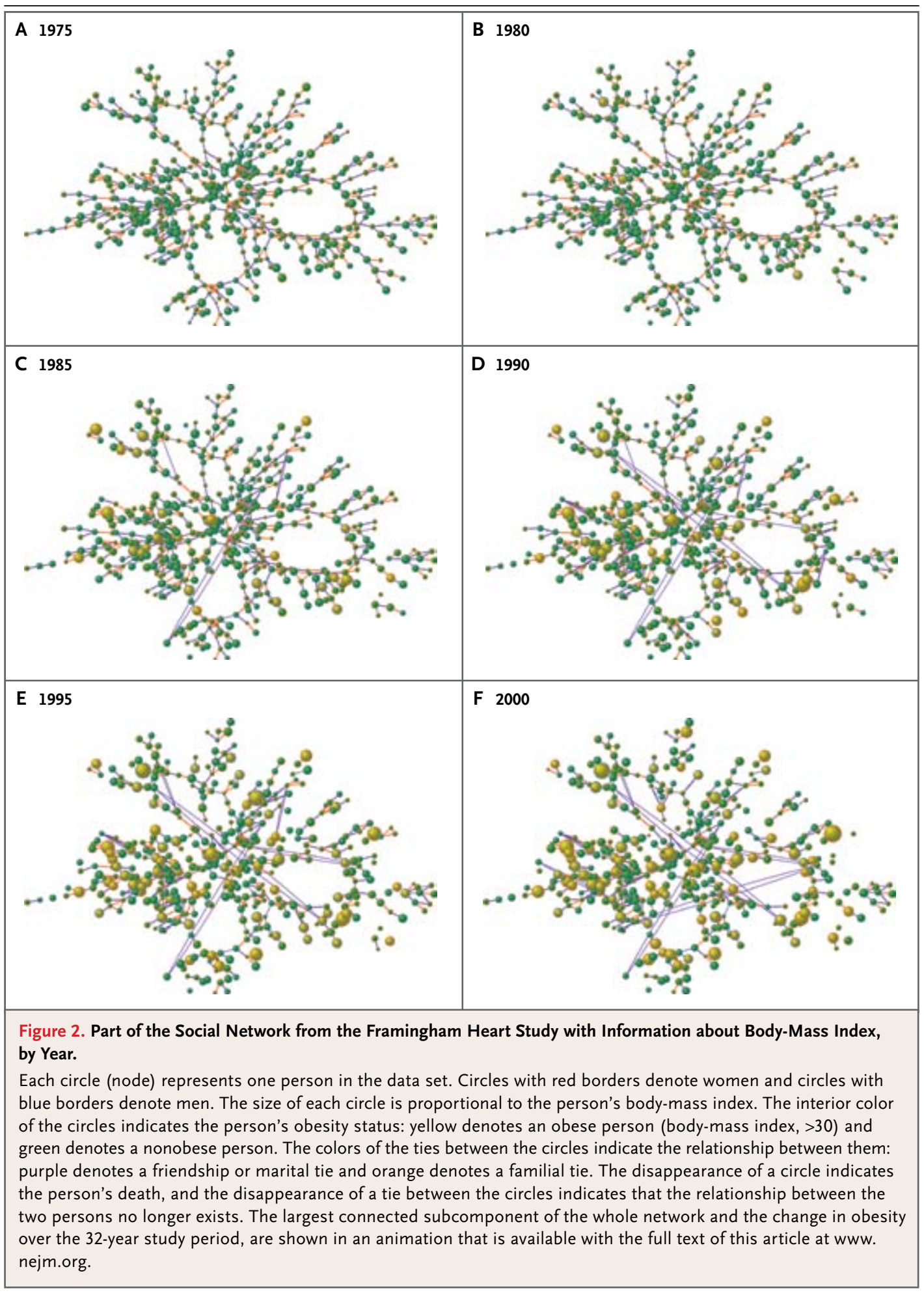


Figure 3. Effect of Social and Geographic Distance from Obese Alters on the Probability of an Ego's Obesity in the Social Network of the Framingham Heart Study.

Panel A shows the mean effect of an ego's social proximity to an obese alter; this effect is derived by comparing the conditional probability of obesity in the observed network with the probability of obesity in identical networks (with topology preserved) in which the same number of obese persons is randomly distributed. The social distance between the alter and the ego is represented by degrees of separation ( 1 denotes one degree of separation from the alter, 2 denotes two degrees of separation from the alter, and so forth). The examination took place at seven time points. Panel B shows the mean effect of an ego's geographic proximity to an obese alter. We ranked all geographic distances (derived from geocoding) between the homes of directly connected egos and alters (i.e., just those pairs at one degree of separation) and created six groups of equal size. This figure shows the average distances for the six mileage groups: 1 denotes 0 miles (i.e., closest to the alter's home), 2 denotes 0.26 mile, 3 denotes 1.5 miles, 4 denotes 3.4 miles, 5 denotes 9.3 miles, and 6 denotes 471 miles (i.e., farthest from the alter's home). There is no trend in geographic distance. I bars for both panels show $95 \%$ confidence intervals based on 1000 simulations. To convert miles to kilometers, multiply by 1.6 .

poraneous obesity (changing from 0 to 1 ), using 1000 randomly drawn sets of estimates from the coefficient-covariance matrix and assuming mean values for all other variables. ${ }^{29}$ All tests were two-tailed. The sensitivity of the results was assessed with multiple additional analyses (see the Supplementary Appendix).

\section{RESULTS}

Figure 1 depicts the largest connected subcomponent of the social network in the year 2000. This network is sufficiently dense to obscure much of the underlying structure, although regions of the network with clusters of obese or nonobese persons can be seen. Figure 2 illustrates the spread of obesity between adjoining nodes in a part of the network over time. A video (available with the full text of this article at www. nejm.org) depicts the evolution of the largest component of the network and shows the progress of the obesity epidemic over the 32-year study period.

Figure 3A characterizes clusters within the entire network more formally. To quantify these clusters, we compared the whole observed net-
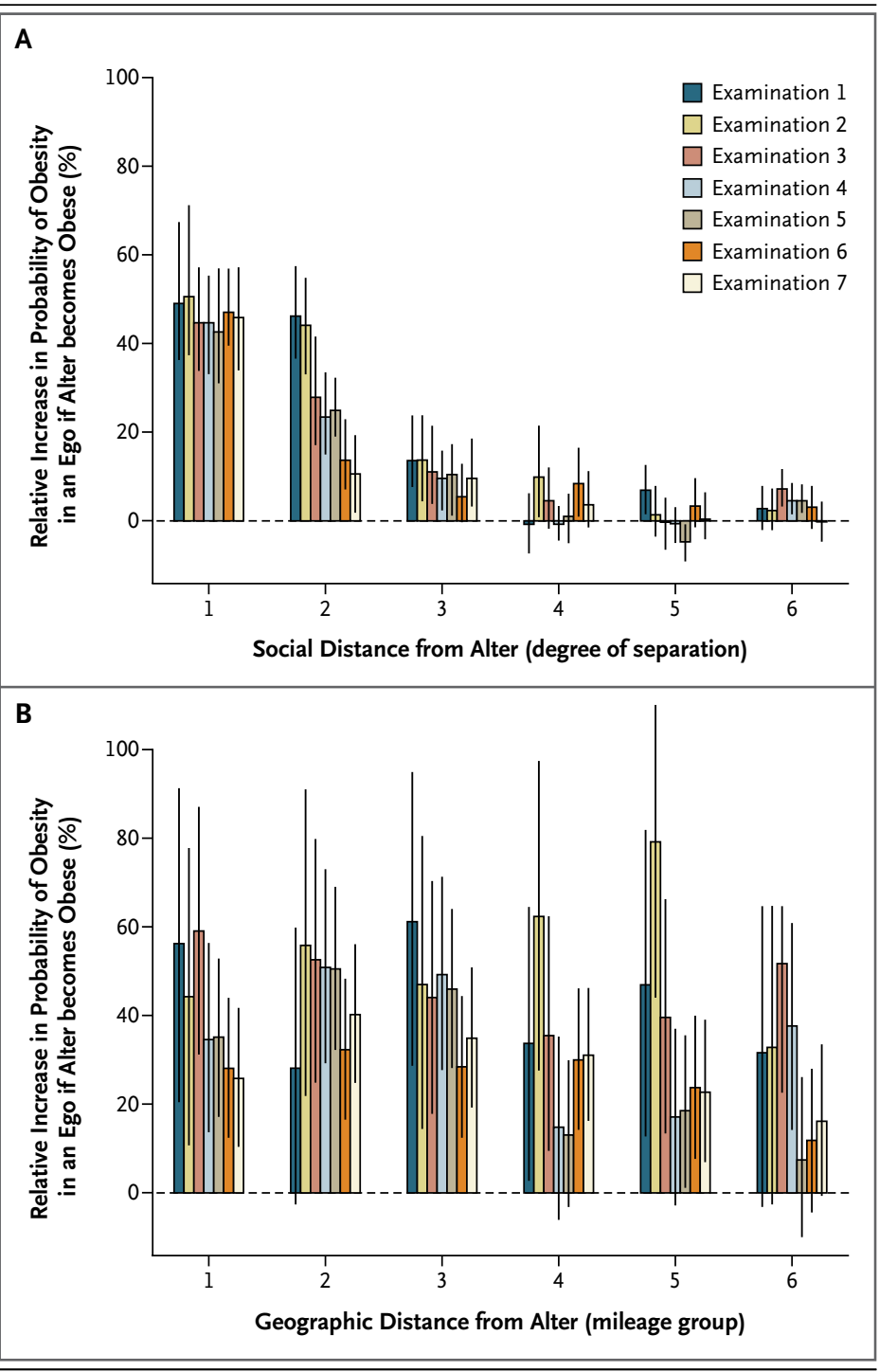

work with simulated networks with the same network topology and the same overall prevalence of obesity as the observed network, but with the incidence of obesity randomly distributed among the nodes (in what we call "random bodymass-index networks"). If clustering is occurring, then the probability that an alter will be obese, given that an ego is known to be obese, should be higher in the observed network than in the random body-mass-index networks. What we call the "reach" of the clusters is the point, in terms of an alter's degree of separation from any given ego, at which the probability of an alter's obesity is no longer related to whether the ego is obese. In all of the examinations (from 1971 through 2003), the risk of obesity among alters 


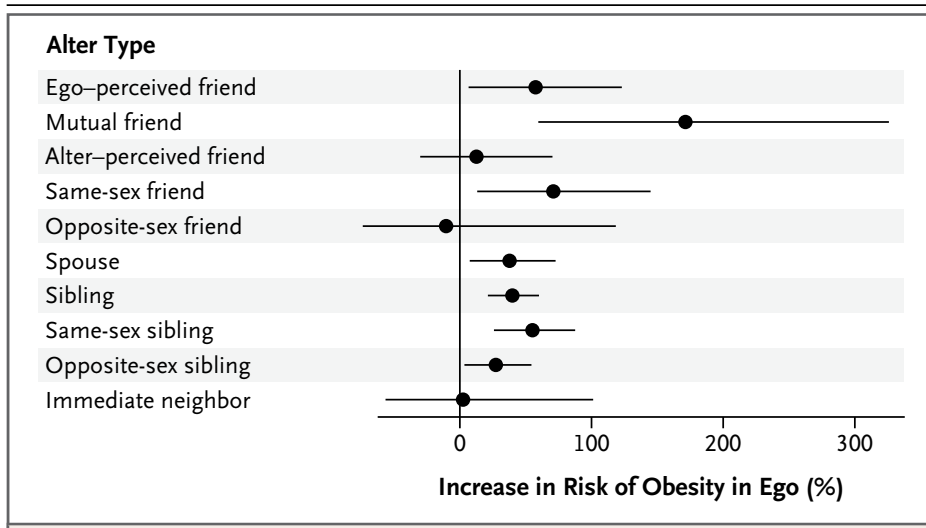

Figure 4. Probability That an Ego Will Become Obese According to the Type of Relationship with an Alter Who May Become Obese in Several Subgroups of the Social Network of the Framingham Heart Study.

The closeness of friendship is relevant to the spread of obesity. Persons in closer, mutual friendships have more of an effect on each other than persons in other types of friendships. The dependent variable in each model is the obesity of the ego. Independent variables include a time-lagged measurement of the ego's obesity; the obesity of the alter; a time-lagged measurement of the alter's obesity; the ego's age, sex, and level of education; and indicator variables (fixed effects) for each examination. Full models and equations are available in the Supplementary Appendix. Mean effect sizes and $95 \%$ confidence intervals were calculated by simulating the first difference in the contemporaneous obesity of the alter (changing from 0 to 1 ) with the use of 1000 randomly drawn sets of estimates from the coefficientcovariance matrix and with all other variables held at their mean values. who were connected to an obese ego (at one degree of separation) was about $45 \%$ higher in the observed network than in a random network. The risk of obesity is also about $20 \%$ higher for alters' alters (at two degrees of separation) and about $10 \%$ higher for alters' alters' alters (at three degrees of separation). By the fourth degree of separation, there was no excess relationship between an ego's obesity and the alter's obesity. Hence, the reach of the obesity clusters was three degrees.

Figure $3 \mathrm{~B}$ indicates that the effect of geographic distance is different from the effect of social distance. Whereas increasing social distance appeared to decrease the effect of an alter on an ego, increasing geographic distance did not. The obesity of the most geographically distant alters correlated as strongly with an ego's obesity as did the obesity of the geographically closest alters. These results suggest that social distance plays a stronger role than geographic distance in the spread of behaviors or norms associated with obesity.

We evaluated the extent of interpersonal association in obesity with the use of regression analysis. Our models account for homophily by including a time-lagged measurement of the alter's obesity. We evaluated the possible role of unobserved contemporaneous events by separately analyzing models of subgroups of the data involving various ego-alter pairings. Figure 4 summarizes the associations.

If an ego stated that an alter was his or her friend, the ego's chances of becoming obese appeared to increase by $57 \%$ ( $95 \%$ confidence interval $[\mathrm{CI}], 6$ to 123) if the alter became obese. However, the type of friendship appeared to be important. Between mutual friends, the ego's risk of obesity increased by $171 \%$ (95\% CI, 59 to 326) if an alter became obese. In contrast, there was no statistically meaningful relationship when the friendship was perceived by the alter but not the ego $(P=0.70)$. Thus, influence in friendship ties appeared to be directional.

The sex of the ego and alter also appeared to be important. When the sample was restricted to same-sex friendships ( $87 \%$ of the total), the probability of obesity in an ego increased by $71 \%$ (95\% CI, 13 to 145) if the alter became obese. For friends of the opposite sex, however, there was no significant association $(\mathrm{P}=0.64)$. Among friends of the same sex, a man had a $100 \%$ (95\% CI, 26 to 197) increase in the chance of becoming obese if his male friend became obese, whereas the female-to-female spread of obesity was not significant (38\% increased chance; $95 \% \mathrm{CI}$, -39 to 161$)$.

Among pairs of adult siblings, one sibling's chance of becoming obese increased by $40 \%$ ( $95 \%$ CI, 21 to 60) if the other sibling became obese. This phenomenon appeared to be more marked among siblings of the same sex $(55 \%$; $95 \%$ CI, 26 to 88) than among siblings of the opposite sex (27\%; 95\% CI, 3 to 54), although the difference was not significant $(\mathrm{P}=0.16)$. Among brothers, an ego's chance of becoming obese increased by $44 \%$ ( $95 \%$ CI, 6 to 91 ) if his alter became obese, and among sisters, an ego's chance of becoming obese increased by $67 \%(95 \% \mathrm{CI}$, 27 to 114) if her alter became obese. Obesity in a sibling of the opposite sex did not affect the chance that the other sibling would become obese.

Among married couples, when an alter became obese, the spouse appeared to be $37 \%$ more likely (95\% CI, 7 to 73 ) to become obese. Husbands and wives appeared to affect each other similarly 
(44\% and 37\%, respectively). Finally, we observed no effect on the risk that an ego would become obese if an immediate neighbor became obese.

We also investigated two factors that might mediate or modify the effect of an alter's weight gain: his or her smoking behavior and geographic distance from the ego (see the Supplementary Appendix). We added measures of smoking behavior for the ego and the alter at both the current and previous examinations. The coefficient for the effect of the alter's obesity was virtually unchanged; smoking behavior does not appear to be instrumental to the spread of obesity. Models that included the geographic distance between the ego and alter corroborated the result shown in Figure 3B: physical distance did not modify the intensity of the effect of the alter's obesity on the ego.

\section{DISCUSSION}

Our study suggests that obesity may spread in social networks in a quantifiable and discernable pattern that depends on the nature of social ties. Moreover, social distance appears to be more important than geographic distance within these networks. Although connected persons might share an exposure to common environmental factors, the experience of simultaneous events, or other common features (e.g., genes) that cause them to gain or lose weight simultaneously, our observations suggest an important role for a process involving the induction and person-to-person spread of obesity.

Our findings that the weight gain of immediate neighbors did not affect the chance of weight gain in egos and that geographic distance did not modify the effect for other types of alters (e.g., friends or siblings) helps rule out common exposure to local environmental factors as an explanation for our observations. Our models also controlled for an ego's previous weight status; this helps to account for sources of confounding that are stable over time (e.g., childhood experiences or genetic endowment). ${ }^{30}$ In addition, the control in our models for an alter's previous weight status accounts for a possible tendency of obese people to form ties among themselves. Finally, the findings regarding the directional nature of the effects of friendships are especially important with regard to the interpersonal induction of obesity because they suggest that friends do not simultaneously become obese as a result of contemporaneous exposures to unobserved factors. If the friends did become obese at the same time, any such factors should have an equally strong influence regardless of the directionality of friendship. This observation also points to the specifically social nature of these associations, since the asymmetry in the process may arise from the fact that the person who identifies another person as a friend esteems the other person.

Finally, pairs of friends and siblings of the same sex appeared to have more influence on the weight gain of each other than did pairs of friends and siblings of the opposite sex. This finding also provides support for the social nature of any induction of obesity, since it seems likely that people are influenced more by those they resemble than by those they do not. Spouses, who share much of their physical environment, may not affect each other's weight gain as much as mutual friends do; in the case of spouses, the opposite-sex effects and friendship effects may counteract each another.

Obesity in alters might influence obesity in egos by diverse psychosocial means, such as changing the ego's norms about the acceptability of being overweight, more directly influencing the ego's behaviors (e.g., affecting food consumption), or both. Other mechanisms are also possible. Unfortunately, our data do not permit a detailed examination. However, some insight into possible mechanisms can be gained from a consideration of the roles of smoking and geographic distance in obesity. The tendency of persons to gain weight when they stop smoking is well known, ${ }^{31}$ and the coincidence of a decrease in smoking and an increase in obesity in the overall population has been noted. ${ }^{32}$ However, the present study indicates that regardless of whether smoking cessation causes weight gain in individual persons, and regardless of whether smoking-initiation or smoking-cessation behavior itself spreads from person to person, ${ }^{33}$ any spread in smoking behavior is not a significant factor in the spread of obesity. This finding indicates that smoking behavior does not mediate the interpersonal effect in the spread of obesity. However, in addition, it suggests that the psychosocial mechanisms of the spread of obesity may rely less on behavioral imitation than on a change in an ego's general perception of the social norms regarding the acceptability of obesity. This point is further 
reinforced by the relevance of the directionality of friendship.

Hence, an ego may observe that an alter gains weight and then may accept weight gain in himself or herself. This weight gain in an ego might, in turn, be determined by various behaviors that an ego chooses to evince, and these behaviors need not be the same behaviors that an alter evinces. The observation that geographic distance does not modify the effect of an alter's obesity also provides support for the concept that norms may be particularly relevant here. Behavioral effects might rely more on the frequency of contact (which one might reasonably expect to be attenuated with distance), whereas norms might not.

The spread of obesity in social networks appears to be a factor in the obesity epidemic. Yet the relevance of social influence also suggests that it may be possible to harness this same force to slow the spread of obesity. Network phenomena might be exploited to spread positive health behaviors, ${ }^{34-36}$ in part because people's percep- tions of their own risk of illness may depend on the people around them. ${ }^{37}$ Smoking- and alcoholcessation programs and weight-loss interventions that provide peer support - that is, that modify the person's social network - are more successful than those that do not. ${ }^{34,35,38,39}$ People are connected, and so their health is connected. ${ }^{40,41}$ Consequently, medical and public health interventions might be more cost-effective than initially supposed, since health improvements in one person might spread to others. ${ }^{42}$ The observation that people are embedded in social networks suggests that both bad and good behaviors might spread over a range of social ties. This highlights the necessity of approaching obesity not only as a clinical problem but also as a public health problem.

Supported by a grant from the National Institutes of Health (NIH R-01 AG24448-01).

No potential conflict of interest relevant to this article was reported.

We thank Laurie Meneades, Rebecca Joyce, Molly Collins, Marian Bellwood, and Karen Mutalik for the expert assistance

required to build the data set, and Emilia Benjamin, Virginia Chang, Scott Desposato, Felix Elwert, Peter Marsden, JoAnne Mu-

rabito, James O'Malley, Barbara McNeil, Mark Pachucki, Mark Pletcher, Mason A. Porter, Darren Schreiber, Richard Suzman, and Alan Zaslavsky for helpful comments.

\section{REFERENCES}

1. Chang VW, Lauderdale DS. Income disparities in body mass index and obesity in the United States, 1971-2002. Arch Intern Med 2005;165:2122-8.

2. Hedley AA, Ogden CL, Johnson CL, Carroll MD, Curtin LR, Flegal KM. Prevalence of overweight and obesity among US children, adolescents, and adults, 19992002. JAMA 2004;291:2847-50.

3. Hill JO, Peters JC. Environmental contributions to the obesity epidemic. Science 1998;280:1371-4.

4. Stunkard AJ, Sorensen TI, Hanis C, et al. An adoption study of human obesity. N Engl J Med 1986;314:193-8.

5. Stunkard AJ, Harris JR, Pedersen NL, McClearn GE. The body-mass index of twins who have been reared apart. $\mathrm{N}$ Engl J Med 1990;322:1483-7.

6. Newman MEJ. The structure and function of complex networks. SIAM Review 2003;45:167-256.

7. Nowak MA, Sigmund K. Evolution of indirect reciprocity. Nature 2005;437: 1291-8.

8. Bearman PS, Moody J, Stovel K. Chains of affection: the structure of adolescent romantic and sexual networks. Am J Sociol 2004;110:44-91.

9. Liljeros F, Edling CR, Nunes Amaral
LA. Sexual networks: implications for the transmission of sexually transmitted infections. Microbes Infect 2003;5:189-96. 10. Watts DJ, Strogatz SH. Collective dynamics of 'small-world' networks. Nature 1998;393:440-2.

11. Sobal J. The size acceptance movement and the social construction of body weight. In: Sobal J, Maurer D, eds. Weighty issues: fatness and thinness as social problems. New York: Aldine de Gruyter, 1999:231-49.

12. Chang VW, Christakis NA. Medical modeling of obesity: a transition from action to experience in a 20th century American medical textbook. Sociol Health Illness 2002;24:151-77.

13. Fogassi L, Ferrari PF, Gesierich B, Rozzi S, Chersi F, Rizzolatti G. Parietal lobe: from action organization to intention understanding. Science 2005;308: 662-7.

14. Whigham LD, Israel BA, Atkinson RL. Adipogenic potential of multiple human adenoviruses in vivo and in vitro in animals. Am J Physiol Regul Integr Comp Physiol 2006;290:R190-R194.

15. Turnbaugh PJ, Ley RE, Mahowald MA, Magrini V, Mardis ER, Gordon JI. An obesity-associated gut microbiome with increased capacity for energy harvest. Nature 2006;444:1027-31.

16. Dawber TR. The Framingham Study: the epidemiology of atherosclerotic disease. Cambridge, MA: Harvard University Press, 1980.

17. Feinleib M, Kannel WB, Garrison RJ,
McNamara PM, Castelli WP. The Framingham Offspring Study: design and preliminary data. Prev Med 1975;4:518-25.

18. Kamada T, Kawai S. An algorithm for drawing general undirected graphs. Information Processing Letters 1989;31:7-15.

19. Batagelj V, Mrvar A. Pajek — analysis and visualization of large networks. In: Junger M, Mutzel P, eds. Graph drawing software. Berlin: Springer, 2003:77-103.

20. Moody J, McFarland DA, BenderdeMoll S. Visualizing network dynamics. Am J Sociol 2005;110:1206-41.

21. Barabasi AL, Albert R. Emergence of scaling in random networks. Science 1999; 286:509-12.

22. Ravasz E, Barabasi AL. Hierarchical organization in complex networks. Phys Rev E Stat Nonlin Soft matter Phys 2003; 67:026112.

23. McPherson M, Smith-Lovin L, Cook JM. Birds of a feather: homophily in social networks. Ann Rev Sociol 2001;27:415-44. 24. Sackett DL, Anderson GD, Milner R, Feinleib M, Kannel WB. Concordance for coronary risk factors among spouses. Circulation 1975;52:589-95.

25. Carrington PJ, Scott J, Wasserman S. Models and methods in social network analysis. New York: Cambridge University Press, 2005.

26. Liang K-Y, Zeger SL. Longitudinal data analysis using generalized linear models. Biometrika 1986;73:13-22.

27. Schildcrout JS, Heagerty PJ. Regression analysis of longitudinal binary data with time-dependent environmental covariates: 
bias and efficiency. Biostatistics 2005;6: 633-52.

28. Beck N. Time-series-cross-section data: what have we learned in the past few years? Annu Rev Polit Sci 2001;4:271-93.

29. King G, Tomz M, Wittenberg J. Making the most of statistical analyses: improving interpretation and presentation. Am J Polit Sci 2000;44:341-55.

30. Atwood LD, Heard-Costa NL, Fox CS, Jaquish CE, Cupples LA. Sex and age specific effects of chromosomal regions linked to body mass index in the Framingham Study. BMC Genet 2006;7:7.

31. Eisenberg D, Quinn BC. Estimating the effect of smoking cessation on weight gain: an instrumental variable approach. Health Serv Res 2006;41:2255-66.

32. Philipson TJ, Posner RA. The long-run growth in obesity as a function of technological change. Perspect Biol Med 2003; 46:Suppl 3:S87-S107.

33. Andrews JA, Tildesley E, Hops H, Li F. The influence of peers on young adult substance use. Health Psychol 2002;21:34957.

34. Wing RR, Jeffery RW. Benefits of recruiting participants with friends and increasing social support for weight loss and maintenance. J Consult Clin Psychol 1999;67:132-8.

35. Malchodi CS, Oncken C, Dornelas EA, Caramanica L, Gregonis E, Curry SL. The effects of peer counseling on smoking cessation and reduction. Obstet Gynecol 2003;101:504-10.

36. Bruckner H, Bearman PS. After the promise: the STD consequences of ado- lescent virginity pledges. J Adolesc Health 2005;36:271-8.

37. Montgomery GH, Erblich J, DiLorenzo R, Bovbjerg DH. Family and friends with disease: their impact on perceived risk. Prev Med 2003;37:242-9.

38. McKnight AJ, McPherson K. Evaluation of peer intervention training for high school alcohol safety education. Accid Anal Prev 1986;18:339-47.

39. Wechsler H, Moeykens B, Davenport A, Castillo S, Hansen J. The adverse impact of heavy episodic drinkers on other college students. J Stud Alcohol 1995;56:628-34. 40. Christakis NA, Allison PD. Mortality after the hospitalization of a spouse. N Engl J Med 2006;354:719-30.

41. Granovetter MS. The strength of weak ties. Am J Sociol 1973;78:1360-80. 